\title{
The influence of microalloying and heat treatment on the struc- ture and properties of Galfenol with high gallium concentration
}

\author{
V. V. Palacheva ${ }^{\dagger, 1}$, V.V. Cheverikin ${ }^{1}$, E. N. Zanaeva ${ }^{1}$, F. Emeis ${ }^{2}$, V. V. Korovushkin ${ }^{1}$, \\ H. Wang ${ }^{3}$, C. Jiang ${ }^{3}$, I. S. Golovin ${ }^{1}$ \\ †lera.palacheva@mail.ru
${ }^{1}$ National university of science and technology "MISiS”, 4 Leninskiy Av., Moscow, 119991, Russia
${ }^{2}$ University of Münster, 2 Schlossplatz, Munster, 48149, Germany
${ }^{3}$ Beihang University, 37 Xueyuan Road, Haidian District, Beijing, 100083, China

\begin{abstract}
The structure of soft-magnetic and magnetostrictive alloys of the Galfenol type Fe-(27-28) at.\% Ga after various heat treatment regimes has been studied. The features of formation of both metastable and equilibrium ordered phases in alloys based on Fe-Ga and Fe-Ga-rare-earth elements have been examined. Alloys of these systems belong to the class of highly magnetostrictive materials (up to 400 ppm for monocrystals) used in electromagnetic drives and special sensors. Two-phase alloys with a nonequilibrium phase based on the bcc lattice ( $\mathrm{D}_{3}$ structure) and the equilibrium phase based on the fcc lattice ( $\mathrm{L}_{2}$ structure) are investigated. Isothermal annealing of the $\mathrm{Fe}_{3} \mathrm{Ga}$ alloy at $400^{\circ} \mathrm{C}$ was used to obtain a microstructure that allows analysis of the kinetics of phase transformations under well-controlled conditions. It is established that isothermal annealing entails the formation of a microstructure with a certain ratio of metastable bcc and equilibrium fcc phases having different magnetic properties. The kinetics and mechanisms of phase transformations in binary Fe-Ga alloys and in multicomponent $\mathrm{Fe}-\mathrm{Ga}-\mathrm{Tb}$ alloys are systematically studied using a complex of methods of physical material science (X-ray diffraction (XRD), scanning electron microscopy with EBSD analysis (SEM-EBSD), optical microscopy (LM), vibration magnetometer (VSM), mössbauer spectroscopy. The influence of microalloying of the alloys with terbium on structure and functional characteristics is discussed. The role of $\mathrm{Tb}$ in the stabilization of nonequilibrium phases at room temperature is shown. The influence of small additions $(0.5$ at.\%) of $\mathrm{Tb}$ on the changes in the structure Fe-Ga alloys was studied by mössbauer spectroscopy.
\end{abstract}

Keywords: Fe-Ga and Fe-Ga-Tb, phase transformations.

УДК: 620.182

\section{Влияние микролегирования и термической обработки на струк- туру и свойства галфенолов с высоким содержанием галлия}

Палачева В. В. ${ }^{\dagger,}$, Чеверикин В. В. ${ }^{1}$, Занаева Э. Н. ${ }^{1}$, Эмеис Ф. ${ }^{2}$, Коровушкин В. В. ${ }^{1}$,

\author{
Ванг Х. ${ }^{3}$, Джанг Ч. ${ }^{3}$, Головин И. С. ${ }^{1}$ \\ †lera.palacheva@mail.ru
}
${ }^{1}$ Национальный Исследовательский Технологический Университет «МИСиС», Ленинский пр., 4, Москва, 119991, Россия ${ }^{2}$ Университет Мюнстера, Шлоссплац, 2, Мюнстер, 48149, Германия
${ }^{3}$ Университет Бейанг, Сюэюань-роуд, 37, Хайдянь, Пекин, 100083, Китай

Исследована структура магнитомягких магнитострикционных сплавов типа Галфенол Fe-(27-28) ат.\% Ga после различных режимов термической обработки. Изучены особенности формирования как метастабильных, так и равновесных упорядоченных фаз в сплавах на основе систем $\mathrm{Fe}-\mathrm{Ga}$ и $\mathrm{Fe}-\mathrm{Ga}-$ редкоземельный элемент. Сплавы указанных систем принадлежат к классу высоко магнитострикционных (до 400 ррm в монокристаллах) материалов, используемых в электромагнитных приводах, гидролокаторах и сенсорах специального назначения. Изучены двухфазные сплавы с неравновесной фазой на основе ОЦК решетки (D0 ${ }_{3}$ структуры) и равновесной фазой на основе ГЦК решет- 
ки ( $\mathrm{L}_{2}$ структура). Изотермический отжиг сплава $\mathrm{Fe} \mathrm{Ga}_{3} \mathrm{Gp} 400^{\circ} \mathrm{C}$ был использован для получения микроструктуры, позволяющей провести анализ кинетики фазовых превращений в хорошо контролируемых условиях. Установлено, что изотермический отжиг влечет образование микроструктуры с определенным соотношением метастабильной ОЦК и равновесной ГЦК фаз, имеющими разные магнитные свойства. В настоящей работе систематически изучены кинетика и механизмы фазовых превращений в двойных Fe-Ga сплавах и в многокомпонентных сплавах Fe-Ga-Tb с использованием комплекса методов физического материаловедения: рентгеновская дифракция (XRD), сканирующая электронная микроскопия с приставкой для EBSD анализа (SEM-EBSD), оптическая микроскопия (LM), вибрационный магнитометр (VSM), мессбауэровская спектроскопия. В работе обсуждается влияние микролегирования сплавов тербием на их структуру и функциональные характеристики. Показана роль Ть в стабилизации неравновесных фаз при комнатной температуре. Методом мессбауэровской спектроскопии изучено влияние малых добавок (до 0.5 ат.\%) тербия на изменения в структуре $\mathrm{Fe}-\mathrm{Ga}$ сплавов.

Ключевые слова: $\mathrm{Fe}-\mathrm{Ga}$ и $\mathrm{Fe}-\mathrm{Ga}-\mathrm{Tb}$, фазовые превращения.

\section{1. Введение}

Сплавы системы Fe-Ga (Галфенолы) являются перспективными магнитострикционными материалами, что обусловлено сочетанием их функциональных и механических свойств. Магнитострикционные материалы, к которым относятся исследуемые сплавы, представляют собой вид функциональных материалов, основной особенностью которых является взаимодействие магнитной и механической энергии при приложении внешнего магнитного поля или напряжения. Явление магнитострикции используется при создании электромагнитных приводов, гидролокаторов, сенсоров и элементов демпфирования [1-3].

Технологические свойства сталей и функциональных сплавов на основе железа в значительной степени зависят от конкретной атомной структуры, объемного содержания фаз, и их микроструктуры. В зависимости от содержания галлия в системе $\mathrm{Fe}-\mathrm{Ga}$ имеет место целый каскад фазовых превращений первого и второго рода, включая возможность образования упорядоченных $\mathrm{L1}_{2}$, D0 ${ }_{3}$ и D0 ${ }_{19}$ структур на основе A1, A2 и А3 фаз с ГЦК, ОЦК и ГП решетками, соответственно. Низкотемпературные диффузионно-контролируемые фазовые превращения в этой системе затруднены, что способствует сохранению при комнатной температуре высокотемпературных фаз, образовавшихся при кристаллизации из расплава [4]. Практически во всех публикациях отмечается, что наилучшие функциональные свойства в Галфенолах достигаются при содержании около 19 или 27 ат.\% Ga, однако эти свойства зависят от режимов обработки сплавов [5] и могут быть улучшены за счет микролегирования редкоземельными элементами [1-3,6-8].

В последнее время значительное внимание уделяется исследованию роли микролегирования $\mathrm{Fe}-\mathrm{Ga}$ сплавов редкоземельными элементами ( $\mathrm{Tb}, \mathrm{Ce}, \mathrm{La}, \mathrm{Er}$ и др.), которые существенно повышают комплекс их свойств и, прежде всего, значения магнитострикции [9-11]. Легирование $\mathrm{Tb}$ приводит к увеличению намагниченности и уменьшению величины поля насыщения. Небольшие добавки тербия повышают константу магнитострикции сплава при комнатной температуре. Влияние легирования редкоземельными элементами на кинетику и последовательность фазовых превращений в системе $\mathrm{Fe}-\mathrm{Ga}$ слабо изучено, известно только о весьма ограниченной их растворимости в твердом растворе, после чего начи- нает выделяться эвтектика, содержащая редкоземельные металлы $[9,10]$. В работах с участием авторов настоящей работы [12,13] отмечается влияние тербия на фазовые превращения в Галфенолах с различным содержанием галлия.

Изложенные факты, функциональные свойства Галфенолов и их относительная дешевизна [10] определяют целесообразность и перспективность систематического изучения структуры и свойств этих сплавов.

Целью данной работы является изучение кинетики и механизмов фазовых превращений в двойных Fe-Ga сплавах, а также исследование влияния микролегирования сплавов тербием на их структуру и функциональные характеристики.

\section{2. Методика проведения исследований}

В качестве объекта исследования использовали двойные и тройные сплавы типа Галфенол на основе систем $\mathrm{Fe}-\mathrm{Ga}$ и $\mathrm{Fe}-\mathrm{Ga}-\mathrm{Tb}$. Содержание $\mathrm{Ga}$ в двойных сплавах варьировалось между 27.0 и 27.8 ат.\% от плавки к плавке, а в тройном оно было 27.4 ат.\% Ga и $0.15 ; 0.5$ aт.\% Tb. Для приготовления сплавов использовали Fе чистотой 99.99\% марки 008ЖР, Ga и Тb чистотой $99.999 \%$. Образцы сплавов получали методом индукционной плавки в печи Indutherm MC-20V. Слиток массой 40 г и размерами $16 \times 4 \times 60$ мм получали литьем в медную изложницу. Микроструктуру сплавов и содержание элементов изучали с помощью энерго-дисперсионной спектроскопии с точностью до \pm 0.1 ат.\% на электронных сканирующих микроскопах Tescan Vega LMH (НИТУ «МИСиС») с приставкой для проведения анализа Oxford Instruments Advanced AZtecEnergy и FEI Nova NanoSEM 230 с приставкой для EBSD анализа (University of Münster). Отжиг образцов проводили в лабораторных печах Nabertherm N 30/65А в интервале температур от 400 до $500^{\circ} \mathrm{C}$ и продолжительностью до 600 минут.

Для изучения фазового состава использовали рентгеновский дифрактометр Bruker D8 Advanced c Cu- $\mathrm{K}_{\alpha 1}$ излучением, с длиной волны $\lambda=1.5406 \AA$ в интервале $2 \Theta$ от 20 до $120^{\circ}$. Намагниченность измеряли на вибрационном магнитометре VSM-130 при температурах до $750^{\circ} \mathrm{C}$ и со скоростью нагрева 6 К/мин, приложенное магнитное поле при измерении составляло $\sim 400$ кА/м.

Измерения мессбауэровской спектроскопии проводили на спектрометре Ms-1104 Em с источником $\gamma$-излу- 
чения $\mathrm{Co}^{57}$ в матрице хрома при комнатной температуре на порошковых пробах. Изомерный сдвиг определяли относительно a-Fe. Математическую обработку измеренных спектров проводили по программе «Univem Ms» (ЮФУ, Ростов-на-Дону). При задании модели учитывали визуальную оценку спектра, возможные минимальную и максимальную ширину резонансных линий в секстетах (от $0.19 \mathrm{~mm} / \mathrm{c}$ до $0.58 \mathrm{~mm} / \mathrm{c}$ ), наличие неэквивалентных положений атомов железа, с различным числом атомов $\mathrm{Ga}$ в ближайшем его окружении и физический смысл результата. Конечный результат обработки оценивали по минимальному значению $\chi^{2}$ (среднеквадратичное отклонение модели от эксперимента). Структуру образцов устанавливали по данным рентгеноструктурного анализа.

\section{3. Результаты исследований и их обсуждение}

\section{1. Макро- и микроструктура слитка}

Слиток двойного сплава $\mathrm{Fe}-27 \mathrm{Ga}$ имеет зону столбчатых кристаллов, растущих от стенки медной изложницы к центру слитка вдоль направления теплоотвода. Крупные, вытянутые в одном направлении кристаллы подавляют рост остальных, менее благоприятно ориентированных кристаллов и имеют предпочтительную кристаллографическую ориентировку - текстуру кристаллизации $<100>$. В поперечном сечении слиток имеет крупные равноосные зерна. В литом состоянии слиток тройного сплава $\mathrm{Fe}-27 \mathrm{Ga}-\mathrm{Tb}$ имеет дендритную микрострук- туру. На Рис. 1a,b видно, что по границе дендритной ячейки образовалась фаза, обогащенная $\mathrm{Tb}$ и $\mathrm{Ga}$ (белый цвет). Гомогенизация сплава по рекомендуемому в работе [11] режиму: $1200^{\circ} \mathrm{C}, 3$ часа в вакууме с последующим охлаждением в кварцевой ампуле приводит к образованию L1 быстрого охлаждения образцов в кварцевой ампуле и, как следствие, к образованию равновесных фаз при охлаждении и коагуляции выделений, обогащенных $\mathrm{Tb}$ и Ga (Рис. 1c).

Результаты калориметрического анализа сплава $\mathrm{Fe}-27.4 \mathrm{Ga}-0.5 \mathrm{~Tb}$ в литом состоянии со скоростью нагрева и охлаждения $20 \mathrm{~K} /$ мин свидетельствуют о возникновении и растворении фазы, обогащенной $\mathrm{Tb}$ и $\mathrm{Ga}$, в интервале температур $1100-1200^{\circ} \mathrm{C}$ (Рис. S1, дополнительный материал). Эти эффекты на кривых тепловыделения отсутствуют в двойном сплаве. При охлаждении тройного сплава наблюдается экзотермический эффект при температуре $1116^{\circ} \mathrm{C}$, связанный с образованием фазы с высоким содержанием Tb и Ga (Табл. 1). Аналогично при нагреве при температуре $1173^{\circ} \mathrm{C}$ регистрируется растворение этой фазы.

\section{2. Распределение элементов $\mathrm{Fe}, \mathrm{Ga}$ u Tb в сплаве $\mathrm{Fe}-27 \mathrm{Ga}-\mathrm{Tb}$}

После литья сплавы Fe-27Ga и $\mathrm{Fe}-27 \mathrm{Ga}-x \mathrm{~Tb}(x=0.15$ и 0.5 ) имеют дендритную структуру. В тройном сплаве обогащенная $\mathrm{Tb}$ и $\mathrm{Ga}$ фаза переменного состава (Рис. S2, дополнительный материал) образуется при кристаллизации по границам зерен и дендритов [14].

Табл. 1. Содержание $\mathrm{Ga}$ и $\mathrm{Tb}$ по сечению дендритной ячейки в литом сплаве $\mathrm{Fe}-27.4 \mathrm{Ga}-0.5 \mathrm{~Tb}$.

Table 1. Concentration of $\mathrm{Ga}$ and $\mathrm{Tb}$ across the section of the dendritic cell in the cast Fe-27.4Ga-0.5Tb alloy.

\begin{tabular}{|c|c|c|c|c|}
\hline \multicolumn{5}{|c|}{ Литое состояние/As-cast state } \\
\hline \multicolumn{2}{|c|}{ Матрица $\left(\mathrm{DO}_{3}\right) /$ Matrix $\left(\mathrm{DO}_{3}\right)$} & \multicolumn{3}{|c|}{$(\mathrm{Tb}+\mathrm{Ga})$ выделения / $(\mathrm{Tb}+\mathrm{Ga})$ phase } \\
\hline $\begin{array}{l}\mathrm{Fe}, \text { aт. } \% \\
\mathrm{Fe}, \text { at.\% }\end{array}$ & $\begin{array}{l}\mathrm{Ga}, \text { aт.\% } \\
\mathrm{Ga}, \text { at. } \%\end{array}$ & $\begin{array}{l}\text { Fe, aт. } \% \\
\text { Fe, at.\% }\end{array}$ & $\begin{array}{l}\mathrm{Ga}, \text { aт. } \% \\
\mathrm{Ga}, \text { at.\% }\end{array}$ & $\begin{array}{l}\mathrm{Tb}, \text { aт. } \% \\
\mathrm{~Tb}, \text { at. } \%\end{array}$ \\
\hline$\sim 73.6$ & $\sim 26.4$ & $\sim 52$ & $\sim 41$ & $\sim 7$ \\
\hline
\end{tabular}

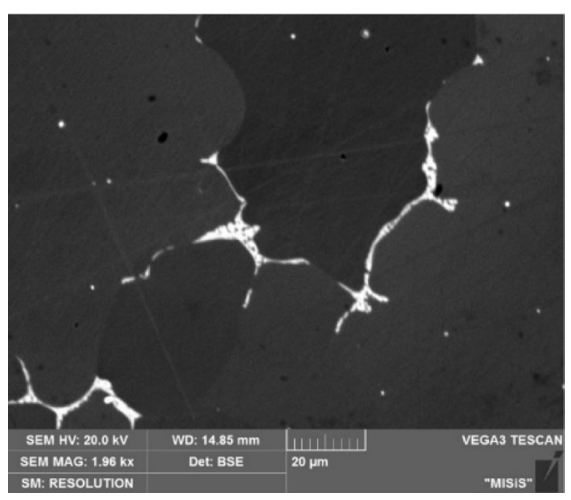

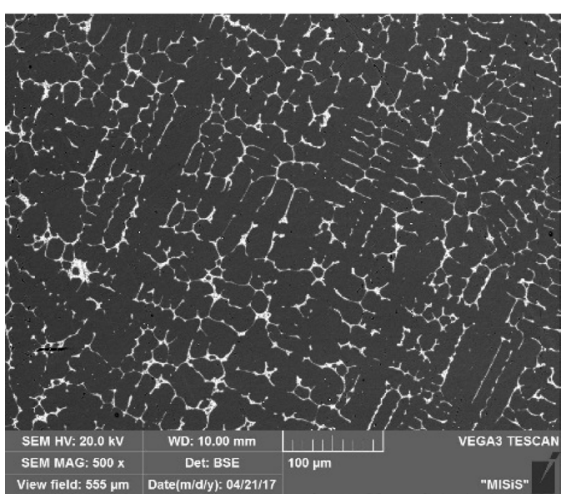

$\mathrm{b}$

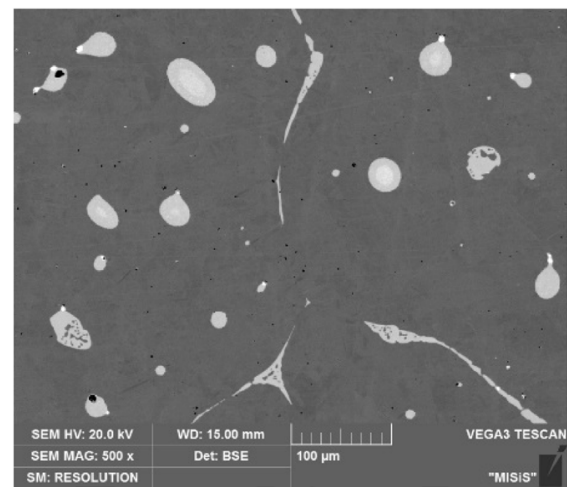

Рис. 1. Микроструктура слитка в литом состоянии составов: Fe-27.4Ga-0.15Tb (a) и Fe-27.4Ga-0.5Tb (b); светлые участки - фаза, одновременно обогащенная $\mathrm{Tb}$ и $\mathrm{Ga}$. Микроструктура гомогенизированного сплава Fe-27.4Ga-0.5Tb при $1200^{\circ} \mathrm{C}, 3$ ч (c).

Fig. 1. Microstructure of the ingot in the cast state: Fe-27.4Ga-0.15Tb (a) and Fe-27.4Ga-0.5Tb (b); bright regions - a phase simultaneously enriched in $\mathrm{Tb}$ and $\mathrm{Ga}$. Microstructure of the homogenized Fe-27.4Ga-0.5Tb alloy at $1200{ }^{\circ} \mathrm{C}, 3 \mathrm{~h}$ (c). 
Как видно из результатов измерения распределения элементов в сплаве Fe-27.4Ga-0.5Tb (Рис. S2, дополнительный материал) фаза переменного состава, образовавшаяся по границе дендритной ячейки при кристаллизации (Рис. S2 a, дополнительный материал), обогащена $\mathrm{Ga}$ до $\sim 41$ ат.\% и Тb до 7 ат.\% (Табл. 1). Линейный размер выделений фазы, обогащенной $\mathrm{Ga}$ и $\mathrm{Tb}$, в литом состоянии достигает 10 мкм. То есть состав фазы переменный и концентрация $\mathrm{Ga}$ и $\mathrm{Tb}$ плавно растет по мере удаления от границы выделения на расстояние приблизительное 10 мкм. Формирование обогащенной Ga и Tb фазы приводит к обеднению матрицы по этим элементам, в частности, наблюдается уменьшение содержания галлия в твердом растворе Fe-Ga сплава почти на $1 \%$ (с 27.4 до 26.4 ат.\%). Такое изменение состава матрицы выводит сплав из однофазной области равновесной диаграммы в двухфазную область равновесной фазовой диаграммы [15]. Аналогичные выделения обогащенной $\mathrm{Ga}$ и $\mathrm{Tb}$ фазы также присутствуют в тройном сплаве c $0.15 \mathrm{~Tb}$, но в значительно меньшем количестве (Рис. 1a), что позволяет грубо оценить растворимость $\mathrm{Tb}$ в $\mathrm{Fe}-\mathrm{Ga}$ как $0.05-0.1 \%$.

На Рис. S2b (дополнительный материал) приведено распределение элементов $(\mathrm{Ga}, \mathrm{Tb}, \mathrm{Fe})$ в матрице и фазе обогащенной $\mathrm{Ga}$ и $\mathrm{Tb}$ в тройном сплаве $\mathrm{Fe}-27.4 \mathrm{Ga}-0.5 \mathrm{~Tb}$ после гомогенизации при $1200^{\circ} \mathrm{C}, 3$ ч. В отличие от литого состояния, в гомогенизированном состоянии, выделения обогащенной $\mathrm{Tb}$ и $\mathrm{Ga}$ фазы, заметно крупнее (более 60 мкм) и имеют в результате процессов коалесценции и коагуляции преимущественно овальную форму. По профилю концентраций можно оценить равновесный состав фазы, обогащенной $\mathrm{Tb}$ и $\mathrm{Ga}$, который достигается по мере удаления от границы фазы на 10 мкм и более, как - $\mathrm{Fe}_{44} \mathrm{Ga}_{47} \mathrm{~Tb}_{9}$. Выявить тип кристаллической решетки этой фазы не удалось.

Исследование сплавов с различным содержанием тербия позволяет сделать вывод, что растворимость $\mathrm{Tb}$ в $\mathrm{Fe}-\mathrm{Ga}$ матрице с $\mathrm{D}_{3}$ структурой составляет менее $0.1 \%$, т. к. в этом сплаве с $0.15 \%$ Tb обогащенная $\mathrm{Ga}$ и $\mathrm{Tb}$ фаза уже отчетливо наблюдается в литом состоянии сплава (Рис. 1a). Наша оценка совпадает с выводами работы [15], в которой авторы также проанализировали концентрационные профили тройного $\mathrm{Fe}-\mathrm{Ga}-\mathrm{Tb}$ сплава. Однако сделать оценку равновесного состава $(\mathrm{Tb}+\mathrm{Ga})$ фазы как $\mathrm{Fe}_{44} \mathrm{Ga}_{47} \mathrm{~Tb}_{9}$ удалось впервые.

\section{3. Влияние Ть на магнитную структуру}

Влияние тербия на повышение магнитострикции в Галфенолах принято связывать с тем, что атомы тербия участвуют в формировании наногетерогенных неоднородностей и усиливают создаваемые ими упругие искажения решетки, которые в свою очередь вносят вклад в деформацию решетки в магнитном поле [17]. Природа наногетерогенностей до конца не исследована, однако она так или иначе связана с образованием пар атомов Ga-Ga в решетке ОЦК железа, то есть с образованием ближнего упорядочения или кластеров с модифицированной $\mathrm{D}_{3}$ структурой [18]. Проведенные нами измерения магнитострикции для сплавов $\mathrm{Fe}-19 \mathrm{Ga}$ и $\mathrm{Fe}-27 \mathrm{Ga}$ с 0.1 и 0.3 ат.\% Тb соответственно подтвердили, что микролегирование $\mathrm{Tb}$ в двойных Fe-Ga сплавах приводит к заметному увеличению значений магнитострикции [19]. Для закаленного сплава $\mathrm{Fe}-27 \mathrm{Ga}$ значение магнитострикции насыщения составило 130 ppm, микролегирование (0.3 ат.\% Тb) тербием привело к увеличению магнитострикции до 240 ppm. Для двойного сплава $\mathrm{Fe}-19 \mathrm{Ga}$ в закаленном состоянии магнитострикция составила 120 ppm, при легировании 0.1 ат.\% Тb значение магнитострикции насыщения возросло до $210 \mathrm{ppm}$.

Сравнение мессбауэровских спектров литых образцов $\mathrm{Fe}-27 \mathrm{Ga} \mathrm{c} \mathrm{Tb}$ и без него, имеющими неравновесную структуру на основе ОЦК решетки, приведены на Рис. S3 (дополнительный материал). Параметры спектров обобщены в Табл. 2. Экспериментальные спектры разложены на шесть секстетов, обеспечивающих минимальное значение среднеквадратичного отклонения модели от эксперимента $\left(\chi^{2}\right)$. Согласно Рис. S3 a (дополнительный материал) и приведенных параметров (Табл. 2), 6 секстетов в спектре литого образца объясняется возникновением неэквивалентных положений атомов железа, в ближайшей координационной сфере которых находится различное число атомов $\mathrm{Ga}$, образовавшееся при кристаллизации сплава. При замещении атомов $\mathrm{Fe}$ атомами $\mathrm{Ga}$, т.е. вследствие уменьшения величины металлической связи между атомами в структуре сплава, происходит обрыв обменных магнитных связей, уменьшение магнитных полей на ядрах $\mathrm{Fe}^{57}$ и увеличение изомерного сдвига.

Анализируя полученные параметры, следует отметить, что даже незначительное содержание легирующего компонента ( 0.5 ат.\% Tb), изменяет такие параметры как: магнитные поля и изомерный сдвиг. Так, начиная со 2-го секстета по 6-ой магнитные поля на ядрах $\mathrm{Fe}^{57}$ в сплаве с добавкой $\mathrm{Tb}$ существенно выше, чем в исходном образце, а изомерный сдвиг, наоборот, уменьшается. Квадрупольный сдвиг практически не изменяется, что указывает на неизменность симметрии $\mathrm{Fe}-\mathrm{Ga}-$ и $\mathrm{Fe}-\mathrm{Ga}-\mathrm{Tb}$-полиэдров. Полученные данные свидетельствуют в пользу гипотезы о формировании наногетерогенных неоднородностей в двойных $\mathrm{Fe}-\mathrm{Ga}$ сплавах за счет микролегирования Тb [17].

\section{4. Влияние отжига на структуру}

Проведено исследование кинетики формирования равновесной структуры при отжиге. Режимы изотермического отжига были выбраны на основе ранее проведенных исследований фазовых превращений в этих сплавах при нагреве методом in situ дифракции нейтронов $[20,21]$ и рентгеновских лучей $[22,23]$. Для структурных исследований был выбран интервал температур изотермического отжига от 400 до $500^{\circ} \mathrm{C}$, который позволяет надежно варьировать и контролировать структуру сплавов. Ha Рис. S4a-c-e (дополнительный материал) приведена структура сплава $\mathrm{Fe}-27 \mathrm{Ga}$ после отжигов различной продолжительности при $400^{\circ} \mathrm{C}$, полученная с помощью SEM-EBSD анализа. Для исследования 
выбрано сечение, перпендикулярное направлению роста столбчатых кристаллов. После отжига при $400^{\circ} \mathrm{C}$ в течение 200 мин структура сплава Fe-27Ga состоит в основном из равноосных зерен (Рис. S4 a, дополнительный материал) с ОЦК решеткой. Твердый раствор атомов $\mathrm{Ga}$ в решетке $\alpha-\mathrm{Fe}$, полученный в результате затвердевания слитков в медной изложнице, не является полностью хаотическим (т.е. не является А2 структурой), а имеет признаки $\mathrm{D0}_{3}$ упорядочения - имеются сверхструктурные отражения в дифракционных спектрах [20,21]. На границе исходных зерен можно заметить первые мелкие зародыши - зерна растущей фазы с ГЦК решеткой (Рис. S4b, дополнительный материал). С увеличением времени отжига до 350 и 600 мин, количество ГЦК фазы растет до приблизительно 10\% и 74\%, соответственно (Табл. 3).

Размер зерен фазы с ГЦК решеткой не равномерен по всей поверхности шлифа, поскольку зерна $\mathrm{L1}_{2}$ фазы (ГЦК) образуются в основном на границах зерен с ОЦК решеткой. При отжиге средний размер ОЦК-фазы уменьшается, а средний размер ГЦК-фазы лишь незначительно увеличивается, увеличение доли $\mathrm{L}_{2}$ фазы происходит в основном не за счет укрупнения сильно фрагментированных зерен, а образования новых, что видно при большем увеличении (Рис. S4, дополнительный материал).

Микролегирование $\mathrm{Fe}-27 \mathrm{Ga}$ сплава тербием существенно влияет на микроструктуру сплава и стабилизирует метастабильную при комнатной температуре $\mathrm{D0}_{3}$ фазу, замедляя образование равновесной $\mathrm{L}_{2}$ фазы как при непрерывном нагреве [24,25], так и при изотермическом отжиге [12-13,20,25].

Как было отмечено выше, фаза, одновременно обогащенная $\mathrm{Tb}$ и $\mathrm{Ga}$, образуется после кристаллизации слитка при температуре около $1120^{\circ} \mathrm{C}$ на границах зерен с ОЦК решеткой и на периферии дендритов (Рис. 1). Таким образом, при последующем отжиге этих сплавов снижается возможность зарождения L1 2 фазы на этих же границах. Кроме того, формирование богатой Tb и Ga фазы на границах, приводит к снижению содержания Ga в твердом растворе (Табл. 1), что также замедляет образование и рост $\mathrm{Ll}_{2}$ фазы.

SEM-EBSD анализ трехфазной структуры $\mathrm{Fe}-27.4 \mathrm{Ga}-0.5 \mathrm{~Tb}$ сплава приведен на Рис. S5 (дополнительный материал). Дисперсность $\mathrm{L1}_{2}$ фазы зави-

Табл. 2. Мессбауэровские параметры образцов: Fe-27Ga и Fe-27,4Ga-0,5Tb.

Table 2. Mössbauer parameters of the samples: Fe-27Ga and Fe-27.4Ga-0.5Tb.

\begin{tabular}{|c|c|c|c|c|c|c|}
\hline $\begin{array}{c}\text { Образец сплава } \\
\text { Fe-Ga } \\
\text { Sample Fe-Ga }\end{array}$ & $\begin{array}{c}\text { Компонента } \\
\text { спектра } \\
\text { Spectrum } \\
\text { Component }\end{array}$ & $\begin{array}{c}\text { Изомерный } \\
\text { сдвиг } \delta, \text { мм/с } \\
\text { Isomeric shift } \delta \text {, } \\
\mathrm{mm} / \mathrm{s}\end{array}$ & $\begin{array}{c}\text { Квадрупольное } \\
\text { расщепление } \\
\Delta, \mathrm{mм} / \mathrm{c} \\
\text { Quadrupole } \\
\text { splitting } \Delta, \\
\mathrm{mm} / \mathrm{s}\end{array}$ & $\begin{array}{c}\text { Магнитные } \\
\text { поля на ядрах } \\
\mathrm{Fe}^{57} H, \text { кЭ } \\
\text { Magnetic fields } \\
\text { on the nuclei } \\
\mathrm{Fe}^{57} \mathrm{H} \text {, kOe }\end{array}$ & $\begin{array}{c}\text { Площади ком- } \\
\text { понент S, \% } \\
\text { Component of } \\
\text { area } S, \%\end{array}$ & $\begin{array}{c}\text { Ширина резо- } \\
\text { нансной линии } \\
\Gamma, \mathrm{mм} / \mathrm{c} \\
\text { The width of the } \\
\text { resonance line } \\
\Gamma, \mathrm{mm} / \mathrm{s}\end{array}$ \\
\hline \multirow{6}{*}{$\mathrm{Fe}-27 \mathrm{Ga}$} & $\mathrm{C} 1$ & 0.08 & 0.02 & 325.9 & 15.4 & 0.52 \\
\hline & $\mathrm{C} 2$ & 0.14 & 0.04 & 297.6 & 27.0 & 0.53 \\
\hline & $\mathrm{C} 3$ & 0.20 & 0.02 & 272.5 & 24.3 & 0.53 \\
\hline & $\mathrm{C} 4$ & 0.22 & 0.04 & 247.5 & 16.0 & 0.47 \\
\hline & C5 & 0.27 & 0.00 & 220.2 & 10.1 & 0.44 \\
\hline & C6 & 0.31 & 0.01 & 182.3 & 7.2 & 0.53 \\
\hline \multirow{6}{*}{$\mathrm{Fe}-27.4 \mathrm{Ga}-0.5 \mathrm{~Tb}$} & $\mathrm{C} 1$ & 0.14 & 0.05 & 319.3 & 16.0 & 0.42 \\
\hline & $\mathrm{C} 2$ & 0.14 & 0.04 & 300.8 & 9.9 & 0.29 \\
\hline & C3 & 0.18 & 0.01 & 283.7 & 37.3 & 0.55 \\
\hline & $\mathrm{C} 4$ & 0.20 & 0.04 & 259.9 & 12.1 & 0.30 \\
\hline & $\mathrm{C} 5$ & 0.22 & 0.01 & 241.1 & 10.7 & 0.41 \\
\hline & C6 & 0.27 & 0.00 & 216.3 & 14.0 & 0.53 \\
\hline
\end{tabular}

Табл. 3. Количество ОЦК и ГЦК фазы в сплаве Fe-27Ga, полученное при EBSD анализе образцов.

Table 3. Amount of bcc and fcc phase in Fe-27Ga alloy, obtained with EBSD analysis of the samples.

\begin{tabular}{|c|c|c|c|c|}
\hline Образец/Sample & $\begin{array}{c}\text { Термическая } \\
\text { обработка } \\
\text { Heat treatment }\end{array}$ & $\begin{array}{c}\text { Время отжига, мин } \\
\text { Time of annealing, min }\end{array}$ & $\begin{array}{c}\text { Количество ОЦК } \\
\text { фазы, \% } \\
\text { Amount of bcc phase, \% }\end{array}$ & $\begin{array}{c}\text { Количество ГЦК } \\
\text { фазы, \% } \\
\text { Amount of fcc phase, \% }\end{array}$ \\
\hline $\begin{array}{c}\text { Образец №1 } \\
\text { Sample 1 }\end{array}$ & $\begin{array}{c}\text { Отжиг } 400^{\circ} \mathrm{C} \\
\text { Annealing } 400^{\circ} \mathrm{C}\end{array}$ & 200 & 99.6 & 0.4 \\
\hline $\begin{array}{c}\text { Образец №2 } \\
\text { Sатрle 2 }\end{array}$ & $\begin{array}{c}\text { Отжиг } 400^{\circ} \mathrm{C} \\
\text { Annealing } 400^{\circ} \mathrm{C}\end{array}$ & 350 & 90.1 & 9.9 \\
\hline $\begin{array}{c}\text { Образец №3 } \\
\text { Sample 3 }\end{array}$ & $\begin{array}{c}\text { Отжиг } 400^{\circ} \mathrm{C} \\
\text { Annealing } 400^{\circ} \mathrm{C}\end{array}$ & 600 & 26.6 & 73.4 \\
\hline
\end{tabular}


сит от температуры отжига: дисперсность $\mathrm{L1}_{2}$ фазы тем выше, чем ниже температура отжига.

Многочисленные центры образования $\mathrm{L1}_{2}$ фазы начинают расти при изотермическом отжиге, образуя равновесную фазу с высокоугловыми границами зерен (угол разориентировки более $15^{\circ}$ ). На Рис. S5a,b высокоугловые границы представлены черными линиями внутри ГЦК фазы, имеющей красный цвет. Согласно спектральному анализу, состав фазы, обогащенной $\mathrm{Tb}$ и $\mathrm{Ga}$ и окруженной $\mathrm{L1}_{2}$ фазой, составляет около 7 ат.\% Tb и 37 ат.\% Ga (желтый цвет на Рис. S5a,b, дополнительный материал).

Наряду со структурными исследованиями были изучены магнитные свойства сплава $\mathrm{Fe}-27 \mathrm{Ga}$ при нагреве и охлаждении. Образец сплава был отожжен при $500^{\circ} \mathrm{C}$ в течение часа. Согласно рентгенофазовому анализу количество $\mathrm{Ll}_{2}$ фазы в этом образце составило $70 \%$. По анализу измерений намагниченности в зависимости от температуры (Рис. S6, дополнительный материал) был сделан вывод о том, что для образца с ГЦК структурой наблюдается эффект сохранения ферромагнетизма до высоких температур - вплоть до $620^{\circ} \mathrm{C}$ при первом нагреве. При этой температуре начинается образование парамагнитной фазы $\mathrm{D}_{19}$ из ферромагнитной $\mathrm{L}_{2}$.

При охлаждении со скоростью $6 \mathrm{~K} /$ мин с $750^{\circ} \mathrm{C}$, то есть из В2 области, до $100^{\circ} \mathrm{C}$ количество $\mathrm{L}_{2}$ фазы увеличивается (т. к. выше $750^{\circ} \mathrm{C}$ фаза $\mathrm{L}_{2}$ не существует) до $30 \%$, оставшееся количество, $70 \%$ высокотемпературной равновесной В2 фазы, при охлаждении приобретает $\mathrm{D0}_{3}$ упорядочение. Поэтому при повторном нагреве этого же образца, намагниченность сначала плавно падает до температуры $460{ }^{\circ} \mathrm{C}$, что связано с потерей ферромагнетизма в $\mathrm{D0}_{3}$ фазе, а затем наблюдается резкий рост намагниченности из-за образования равновесной ферромагнитной $\mathrm{L1}_{2}$ фазы. При температуре выше $620^{\circ} \mathrm{C}$ при повторном нагреве намагниченность вновь падает из-за вновь образования парамагнитной D0 ${ }_{19}$ фазы.

\section{4. Заключение}

C помощью сканирующей электронной микроскопии с использованием EBSD анализа, вибрационной магнитометрии и мессбауэровской спектроскопии изучены фазовые переходы и структура литых сплавов $\mathrm{Fe}-(27-28)$ ат.\% $\mathrm{Ga}$ с и без дополнительного микролегирования тербием (до 0.5 ат.\%). $\mathrm{C}$ помощью изотермического отжига литого $\mathrm{Fe}-\mathrm{Ga}$ сплава сформирована микроструктура с различным соотношением метастабильной ОЦК и равновесной ГЦК фаз, имеющими разные магнитные свойства. Выявлено влияние $\mathrm{Tb}$ на структуру Fe-27Ga сплава, показана возможность стабилизации метастабильной $\mathrm{D0}_{3}$ фазы за счет микролегирования тербием. В тройном сплаве наблюдается конкуренция между зарождением и ростом $\mathrm{L}_{2}$ фазы и фазы обогащенной $\mathrm{Tb}$ и $\mathrm{Ga}$, по границам дендритов и зерен $\mathrm{D0}_{3}$ фазы.
Благодарности/Acknowledgements. Авторы выражают благодарность к.т.н. Чурюмову А.Ю. за помощь в производстве сплавов и проведении калориметрических исследованиц, инженеру Базлову И.В. за изготовление образиов для исследований и к. бб-м.н. Морченко А. Т. за дискуссию. Работа выполнена при поддержке РФФИ грант №18-58-53032, раздел по мессбауэровской спектроскопии выполнен при поддержке гранта РНФ №18-12-00283./The authors are grateful to Dr. Churyumov A. Yu. for assistance in the production alloys and calorimetric research, engineer Bazlov I. V. for preparing samples for research and Dr. Morchenko A. T. for discussion. This work was supported by the Russian Foundation for Basic Research grant No. 18-58-53032, the section mössbauer spectroscopy was supported by the grant of the Russian Science Foundation No. 18-12-00283.

Дополнительный материал/Supplementary Material. Электронная версия статьи содержит дополнительный материал (рисунки), доступный безвозмездно на сайте журнала (lettersonmaterials.com)./The online version of this paper contains supplementary material (figures) available free of charge at the journal's Web site (lettersonmaterials.com).

\section{Литература/References}

1. A.E. Clark, J. B. Restorff, M. Wun-Fogle, T. A. Lograsso, D. L. Schlagel. IEEE Trans Magn. 36 (5), 3238 (2000).

2. A.E. Clark, M. Wun-Fogle, J.B. Restorff, T. A. Lograsso, J. R. Cullen. IEEE Trans Magn. 37 (4), 2678 (2001).

3. G. W. Smith, J. R. Birchak. J Appl. Phys. 39 (5), 2311-5 (1968).

4. Q.Z. Chen, A.H.W. Ngan, B.J. Duggan. Journal of Materials Science. 33, 5405 (1998).

5. I. V. Gervasyeva, V. A. Milyutin. Letters on Materials. 8 (3) 341 (2018). DOI: 10.22226/2410-3535-2018-3-341-345

6. A.E. Clark, K. B. Hathaway, M. Wun-Fogle, J.B. Restorff, T. A. Lograsso, T. A. Keppens et al. J Appl Phys. 93 (10), 8621 (2003).

7. E. M. Summes, T. A. Lograsso, M. Wun-Fogle. Journal of Materials Science. , 42, 9582 (2007).

8. W. Wu, J. Liu, C. Jiang. Journal of Alloys and Compounds. 622, 379 (2015).

9. Z. Yao, X. Tian, L. Jiang et al. Journal of Alloys and Compounds. 637, 431 (2015).

10. T. Ma, S. Hu, G. Bai. Applied Physics Letters. 10, 112401 (2015).

11. X. Ren. Phys. Status Solidi B. 251 (10) 1982 (2014).

12. A. Emdadi, V. V. Palacheva, V. V. Cheverikin, S. Divinski, G. Wilde, I. S. Golovin. Journal of Alloys and Compounds. 758, 214 (2018).

13. A. Emdadi, V.V. Palacheva, A.M. Balagurov, I. A. Bobrikov, V.V. Cheverikin, J. Cifre, I.S. Golovin. Intermetallics. 93, 55 (2018).

14. I. S. Golovin, A. M. Balagurov, V. V. Palacheva, A. Emdadi, I. A. Bobrikov, V.V. Cheverikin, A.S. Prosviryakov, S. Jalilzadeh. Journal of Alloys and Compounds. 751364 (2018).

15. O. Kubaschewski. Iron-binary phase diagrams. Berlin, Springer Verlag (1982) 182 p. 
DOI: $10.1007 / 978-3-662-08024-5$

16. Y. Han, H. Wang, T. Zhang, Y. He, C. Jiang. Scripta Materialia. 150, 101 (2018).

17. Y. He, C. Jiang, W. Wu, B. Wang, H. Duan, H. Wang, T. Zhang, J. Wang, J. Liu, Z. Zhang, P. Stamenov, J. M. D. Coey, H.Xu. Acta Materialia. 109, 177 (2016).

18. C. Menga, Y. Wua, C. Jiang. Materials \& Design. 130183 (2017).

19. I.S. Golovin, A.M. Balagurov, V.V. Palacheva, A. Emdadi, I.A. Bobrikov, A. Yu Churyumov, V. V. Cheverikin, A. V. Pozdniakov, A. V. Mikhaylovskaya, S. A. Golovin. Journal of Alloys and Compounds. 707, 51 (2017).

20. I. S. Golovin, A. M. Balagurov, V. V. Palacheva, I. A. Bobrikov,
V. B Zlokazov. Materials and Design. 98, 113 (2016).

21. I.S. Golovin, A.M. Balagurov, I.A. Bobrikov, V. V. Palacheva, J. Cifre. Journal of Alloys and Compounds. 675, 393 (2016).

22. I. S. Golovin, V. V. Palacheva, A. I. Bazlov, J. Cifre, J. Pons. Journal of Alloys and Compounds. 644, 959 (2015).

23. I.S. Golovin, V.V. Palacheva, A.I. Bazlov, J. Cifre, N. Nollmann, S.V. Divinski, G. Wilde. Journal of Alloys and Compounds. 656, 897 (2016).

24. V.V. Palacheva, A. Emdadi, F. Emeis, I. A. Bobrikov, A. M. Balagurov, S. V. Divinski, G. Wilde, I.S. Golovin. Acta Materialia. 130, 229 (2017).

25. F. Gao, C. Jiang, J. Liu, and H. Xu. J. Appl. Phys. 100, 123916 (2006). 